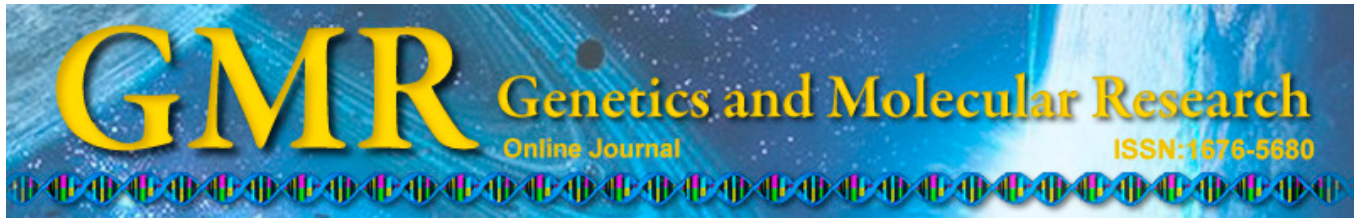

\title{
A promoter trap vector for knocking out bovine myostatin gene with high targeting efficiency
}

\author{
L.H. Zhao ${ }^{1,3 *}$, Y.H. Zhao ${ }^{1 *}$, H. Liang ${ }^{1}$, T. Yun ${ }^{1}$, X.J. Han ${ }^{1}$, M.L. Zhang ${ }^{2}$, \\ X. Zhou ${ }^{1}$, D.X. Hou ${ }^{1}$, R.F. Li ${ }^{2,3}$ and X.L. Li ${ }^{1}$ \\ ${ }^{1}$ The Key Laboratory of National Education Ministry for Mammalian \\ Reproductive Biology and Biotechnology, Inner Mongolia University, \\ Hohhot, China \\ ${ }^{2}$ State Key Laboratories of Reproductive Medicine, \\ Nanjing Medical University, Nanjing, China \\ ${ }^{3}$ Jiangsu Key Laboratory of Xenotransplantation, \\ Nanjing Medical University, Nanjing, China \\ *These authors contributed equally to this study. \\ Corresponding authors: R.F. Li / X.L. Li \\ E-mail: lirongfeng@njmu.edu.cn / lixueling@hotmail.com
}

Genet. Mol. Res. 14 (1): 2750-2761 (2015)

Received February 21, 2014

Accepted August 21, 2014

Published March 31, 2015

DOI http://dx.doi.org/10.4238/2015.March.31.5

\begin{abstract}
With the development of gene targeting approaches, genomic mutation technologies in livestock animals such as gene trapping, zinc finger nucleases (ZFNs), transcription activator-like effector nucleases, and clustered regularly interspaced short palindromic repeats and their associated systems have been improved. Although ZFNs have been used for gene targeting in many species, the off-target sites are still present. Using gene trapping, the workload of screening of targeted clones was decreased by generating a smaller number of drugresistant clones. Determining whether the efficiency of gene trapping is lower than that of ZFNs for a specific gene has been challenging. In this study, to knock out the bovine myostatin gene, we constructed a promoter trap vector and compared its efficiency with that of ZFNs.
\end{abstract}


The promoter trap vector contained a green fluorescent protein sequence without the promoter and a neomycin phosphotransferase $\left(\mathrm{neo}^{\mathrm{R}}\right)$ cassette driven by the phosphoglycerate kinase promoter. When the trapping vector was inserted downstream of the endogenous promoter, the fluorescent protein gene was expressed. The targeted-positive cell clones were identified based on green fluorescence and G418 double selection, followed by polymerase chain reaction analysis and sequencing. The targeting efficiency reached $5 \%$. Compared with the efficiency of ZFN pairs (5.17 and 2.86\%), the promoter trap vector PIII-myostatin could knock out the bovine myostatin gene. Therefore, gene trapping may be an effective tool for genomic modification.

Key words: Bovine fetal fibroblasts; Knock out; Myostatin; Promoter trap vector; Zinc figure nucleases

\section{INTRODUCTION}

Gene targeting is the most effective means of assessing gene function and producing targeted animals. Gene targeting is achieved through homologous recombination between endogenous gene loci and targeting vectors introduced into cells. Although very large numbers of homologous recombinants have been over the past 30 years, a comparatively low rate of exogenous DNA has been introduced into cells and integrated into the genome, which is the primary bottleneck of gene targeting. Various approaches have been successfully used for gene targeting, such as increasing the target cell population, the use of vectors carrying reporter genes, changing the means of DNA delivery, and using recombinant adeno-associated viruses (Sedivy and Dutriaux, 1999; Porteus and Baltimore, 2003). However, powerful functional genomic technologies for mutating genes in livestock animals, such as gene trapping (Marques et al., 2006), zinc finger nucleases (ZFNs) (Hauschild et al., 2011), and transcription activator-like effector nucleases (TALENs) (Carlson et al., 2012a) have also been developed to drastically improve gene targeting.

Recently, gene-targeting approaches have been developed using site-specific nucleases with fusions between the DNA cleavage domain of FokI and a custom-designed DNA-binding domain. ZFNs have $\mathrm{C} 2 \mathrm{H} 2$ zinc-finger motifs, TALENs have truncated transcription activatorlike effector domains, and both induce double-stranded breaks at desired loci that can be repaired by error-prone non-homologous end-joining to yield small insertions and deletions at break sites (Carroll, 2011; Miller et al., 2011). ZFNs have become the most powerful tools for inducing mutations in any locus of any genome. However, the generation of custom ZFNs targeting a desired sequence with high specificity and activity remains challenging, primarily because the fingers for some nucleotide triplets and the context effects of individual fingers in an array are generally unknown (Bogdanove and Voytas, 2011). In addition, few studies have reported the successful design and construction of ZFNs, limiting the cost-effectiveness of this technique.

Gene trapping is an attractive mutagenesis strategy that includes a splice acceptor site immediately upstream of a selectable marker or a reporter gene that lacks a promoter or polyA. This system provides expression data by creating a fusion transcript between a splice donor of an endogenous gene and the splice acceptor of a reporter gene (Friedrich and Soriano, 1991; Zambrowicz et al., 1998). Because the selectable marker in these vectors lacks a promoter sequence or polyA signal, they are particularly effective when combined with homology arms and used for 
gene targeting (i.e., 'targeted trapping') (Friedel et al., 2005). Gene trapping vectors can generate a smaller number of reporter gene-positive clones; however, in many cases, a high proportion of these clones are found resulted from homologous integration, thereby decreasing the workload of screening the targeted clones. In addition, the high targeting efficiency of promoter-trap constructs shows limited versatility. The use of a promoter trap requires the disruption of an exon and the insertion of a selectable marker, expressed either as an in-frame fusion with the endogenous gene or in a separate cistron using an internal ribosomal entry site. Because it is difficult to avoid the loss of gene function resulting from disruption of an endogenous exon, the gene-trapping strategy is more effective for mutations and deletions of the targeting sequence. Thus, as an efficient method for assembling genetic constructs by homologous recombination and promoter trapping with the approximate efficiency of gene targeting to ZFNs is highly desired.

In this study, we constructed a promoter trap vector to knock out the bovine myostatin $(M S T N)$ gene and verified the effectiveness of gene targeting using this vector. The advantages of gene trapping were compared to those of ZFNs.

\section{MATERIAL AND METHODS}

\section{Vector preparation}

\section{Promoter trap vector construction}

Expression of the MSTN gene in bovine fetal fibroblasts was detected by reverse transcription-polymerase chain reaction (PCR) analysis and sequencing (Zhao et al., 2012). The promoter trap vector PIII-MSTN was constructed to knock out the MSTN gene in bovine primary fetal fibroblasts. Regions of homology at MSTN loci were amplified from genomic DNA obtained from ear tissue of Inner Mongolia local black cattle by PCR. Unique restriction sites were added to the primer pairs used to amplify all plasmid components. The PCR-amplified short homology arm was then digested with HindIII and ligated into the PIII plasmid so that promoter-free green fluorescent protein (GFP) could be translated in-frame. Finally, the PCR-amplified long homology arm was inserted downstream of the neomycin phosphotransferase $\left(n^{\mathrm{R}} \mathrm{o}^{\mathrm{R}}\right)$ reading frame of PIII by SacII digestion. The PIII plasmid was a functional vector containing the GFP sequence without a promoter and the $\mathrm{neo}^{\mathrm{R}}$ cassette driven by the phosphoglycerate kinase promoter. The schematic for targeting the MSTN gene in bovine by PIII-MSTN is shown in Figure 1.

\section{Design and construction of ZFNs}

To knockout the second exon of the bovine MSTN gene, 2 pairs of ZFN plasmids (respectively referred to as ZFNs-MSTN I and ZFNs-MSTN II) were designed and assembled by Sigma-Aldrich (St. Louis, MO, USA) (Figure 2). The ZFNs contain the cytomegalovirus promoter, zinc finger modules, FokI domain, bovine growth hormone polyA, Kana ${ }^{\mathrm{R}}$, and pUC origin of replication. Full-ZFN sequences are provided in figures.

\section{Preparation of fibroblasts}

Bovine primary fetal fibroblasts were isolated from fetuses on day 50 of gestation of Inner Mongolia local black cattle. Fetal muscle tissues were minced and dissociated in Dul- 


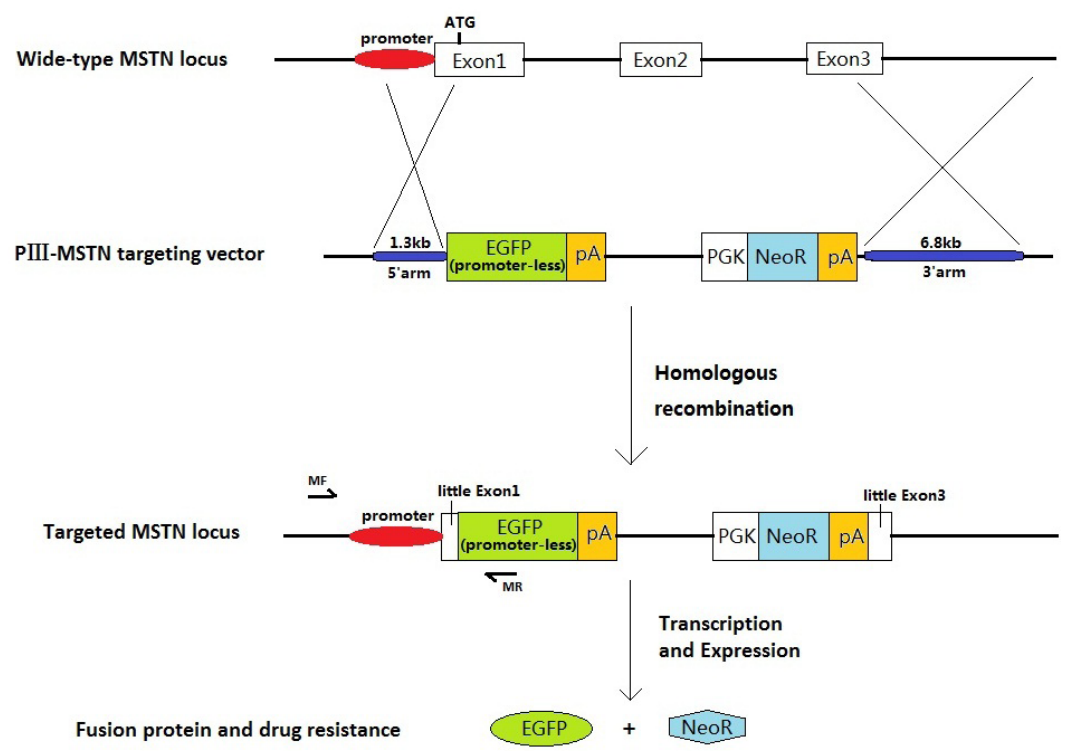

Figure 1. Schematic of bovine myostatin $(M S T N)$ gene trapping using the PIII-MSTN vector. Bovine MSTN genomic locus is shown together with the vector-integration site (blue bars show homologous arms). When the wild-type MSTN was targeted through homologous recombination between the endogenous gene locus and the targeting vector, the targeted locus was an MSTN knockout sequence containing a minimal promoter upstream of the MSTN gene (red ellipse), the reporter gene EGFP without the promoter (green box), a selectable gene $\mathrm{Neo}^{\mathrm{R}}$ expression unit, and some small exons (white boxes). While the fusion protein (EGFP) and drug resistance $\left(\mathrm{Neo}^{\mathrm{R}}\right.$ ) were transcribed and translated at the same time, the MSTN knockout was detected by PCR analysis and sequencing. MF and MR (black arrows) indicate the PCR primers used in PCR analysis and sequencing.

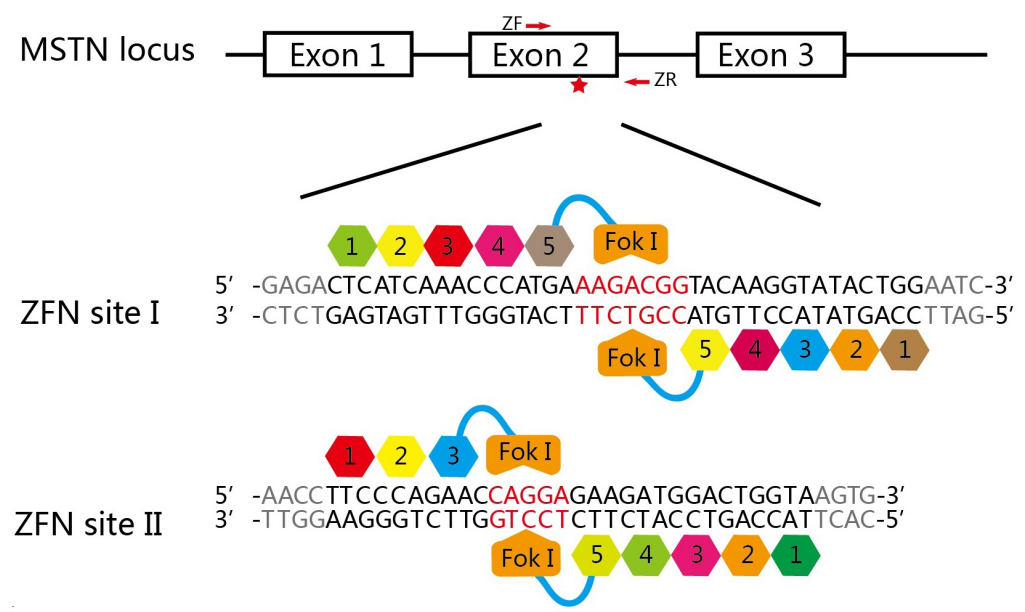

Figure 2. Target sites of ZFNs. The target sites of 2 ZFN plasmids (ZFNs-MSTN I and ZFNs-MSTN II) are both located at the second exon of the bovine MSTN gene. Black boxes and the red star show the exons of bovine MSTN gene and the position of the ZFN-targeted site, respectively. The magnified view illustrates binding sites for the ZFN pairs, and all ZFN variants were based on 3-5 zinc-finger modules, the inter-domain linker, and the FokI cleavage domain. ZF and ZR (red arrows) indicate the PCR primers used in sequence analysis. 
becco's modified Eagle's medium (DMEM) supplemented with $0.53 \mathrm{mg} / \mathrm{mL}$ collagenase and $25 \mathrm{~K} \mathrm{U} / \mathrm{mL}$ DNase I (Sigma) for $2-3 \mathrm{~h}$ at $38.5^{\circ} \mathrm{C}$. The dissociated cells were washed once in DMEM supplemented with 10\% (v/v) fetal bovine serum (FBS) (Hyclone, Logan, UT, USA) by centrifugation at $400 \mathrm{~g}$ for $5 \mathrm{~min}$, and subsequently seeded on $100-\mathrm{mm}$ plastic culture dishes. Seeded cells were cultured for 3-4 days in high-glucose DMEM supplemented with $10 \%(\mathrm{v} / \mathrm{v}) \mathrm{FBS}$ in a humidified incubator with $5 \% \mathrm{CO}_{2}$. Attached cells were further cultured until confluence was reached, trypsinized for 3 min using $0.05 \%$ trypsin, and then stored in freezing medium in liquid nitrogen. The freezing medium consisted of $10 \%(\mathrm{v} / \mathrm{v})$ dimethyl sulfoxide (Sigma) and 90\% (v/v) FBS.

\section{Gene transfection}

\section{Comparison of gene transfection}

Because gene transfection is one of the most critical determinants of gene targeting, we first tested the transfection efficiency of electroporation and lipofection in bovine fetal fibroblasts using the GFP expression vector EGFP-C1. The plasmid EGFP-C1 was linearized by the restriction enzyme SalI and transfected into cells using 2 methods. GFP-positive cells were analyzed by fluorescence microscopy and flow cytometric analysis after a 48 -h culture in a humidified incubator with $5 \% \mathrm{CO}_{2}$ at $38.5^{\circ} \mathrm{C}$.

\section{Electroporation}

The electroporation method was used to introduce the promoter trap vector PIIIMSTN to bovine fetal fibroblasts. Prior to transfection, fibroblasts were thawed and cultured for 3-4 days until confluency, unattached from the culture dishes by trypsinization for $3 \mathrm{~min}$, and cells were counted using a hemocytometer. Cells were harvested by centrifugation at 400 $g$ for $5 \mathrm{~min}$ and resuspended at $1 \times 10^{7} / \mathrm{mL}$ in DMEM/F12 (Invitrogen, Carlsbad, CA, USA) at $0^{\circ} \mathrm{C}$ for transfection. The plasmids PIII-MSTN had been linearized by the restriction enzyme ClaI. The linearized PIII-MSTN and EGFP-C1 were respectively extracted with phenol and phenol/chloroform $(1: 1, \mathrm{v} / \mathrm{v})$ and precipitated with ethanol, and then resuspended at $2 \mu \mathrm{g} / \mathrm{mL}$ in DMEM/F12. Next, $0.4-0.6-\mathrm{mL}$ aliquots of the cell suspension and $10 \mu \mathrm{g}$ linearized plasmid were transferred to 4-mm electroporation cuvettes (Bio-Rad, Hercules, CA, USA), gently mixed, and incubated on ice for $3 \mathrm{~min}$. The cuvette was placed into an electroporation apparatus (BTX, Holliston, MA, USA) at room temperature and electroporated at $400 \mathrm{~V}$ for $4 \mathrm{~ms}$. The cuvette was placed on ice and incubated for another $3 \mathrm{~min}$, and all transfected cells were washed in fresh DMEM with 10\% (v/v) FBS and seeded on 100-mm culture dishes.

\section{Lipofection}

The lipofection method was used to transfect bovine fetal fibroblasts with ZFN plasmids. Fibroblasts were thawed and seeded on 24-well plates in DMEM containing 10\% (v/v) FBS. Cells at $60-70 \%$ confluence were transfected for $4-5 \mathrm{~h}$ with $800 \mathrm{ng}$ ZFN plasmids and linearized EGFP-C1 using Lipofectamine ${ }^{\circledR} 2000$ Reagent (Invitrogen), respectively, according to manufacturer recommendations. After $24 \mathrm{~h}$, the cells were trypsinized and seeded again on 96-well plates at 1 cell per well. After culturing for 8-10 days, colonies were expanded on 
24-well plates; half of the cells were used for PCR analysis and half of the cells were stored in freezing medium in liquid nitrogen.

\section{Screening of targeting cell clones}

\section{G418 screening}

G418 selection ( $800 \mu \mathrm{g} / \mathrm{mL}$; Invitrogen) was applied after $24 \mathrm{~h}$ of culturing the transfected cells. After 7-10 days, resistant cell clones with green fluorescence were screened under a fluorescence microscope (Nikon, Tokyo, Japan) and single clones were placed on 48-well plates, then expanded for PCR analysis or stored in freezing medium in liquid nitrogen for nuclear transfer.

\section{PCR screening and sequencing analysis}

To detect homologous recombination in G418-resistant green fluorescence cell clones, PCR across the short homologous junction and sequencing analysis was conducted using the transgene-specific primers MF and MR. Positive clones were confirmed by PCR amplification across the short homologous arm. The locations of PCR primers are shown in Figure 1. Genomic DNA was extracted from single clones grown on 48-well plates using cell lysis buffer $(100 \mathrm{mM}$ Tris- $\mathrm{HCl}, \mathrm{pH} 8.0,200 \mathrm{mM} \mathrm{NaCl}, 5 \mathrm{mM}$ ethylenediamine tetraacetic acid, $\mathrm{pH} 8.0,0.2 \%$ sodium dodecyl sulfate, $200 \mu \mathrm{g} / \mathrm{mL}$ proteinase $\mathrm{K})$. PCR amplification using the primer pair MF: 5'-TATCCAACTCCAGGACCTGAGATCCCAATT-3' and MR: 5'-AGTTCACCTTGATGCCGTTCTTCTGCTTGT-3' was carried out for 35 cycles with denaturing at $95^{\circ} \mathrm{C}$ for $50 \mathrm{~s}$, annealing at $64^{\circ} \mathrm{C}$ for $50 \mathrm{~s}$, extension at $72^{\circ} \mathrm{C}$ for $3 \mathrm{~min}$, and a final extension at $72^{\circ} \mathrm{C}$ for $10 \mathrm{~min}$. PCR products were analyzed by agarose gel electrophoresis, gel-purified using the SV Gel and PCR Clean-Up System (Promega, Madison, WI, USA) as per manufacturer recommendations, cloned into the PMD ${ }^{\mathrm{TM}}$ 19-T vector (Takara, Shiga, Japan), and sequenced by the Biological Sciences Sequencing Service of Invitrogen. All DNA sequence queries were performed using the alignment tool in CLC Sequence Viewer 6.3.

ZFN knockout events were also detected by PCR and sequencing. Genomic DNA from colonies on 24-well plates was extracted using the Wizard ${ }^{\circledR}$ Genomic DNA Purification Kit (Promega). PCR amplification using the primer pair ZF: 5'-GATTGATATGGAGGTGTTCGTT-3' and ZR: 5'-ACTAGAATCCACTGTGAAGACT-3' was carried out for 35 cycles with denaturing at $95^{\circ} \mathrm{C}$ for $30 \mathrm{~s}$, annealing at $54^{\circ} \mathrm{C}$ for $30 \mathrm{~s}$, extension at $72^{\circ} \mathrm{C}$ for $30 \mathrm{~s}$, and a final extension at $72^{\circ} \mathrm{C}$ for $10 \mathrm{~min}$. PCR products were sequenced using the primer $\mathrm{ZR}$ by the Biological Sciences Sequencing Service of Invitrogen. Fragments corresponding to mutated alleles (locus between ZF and ZR in Figure 2) were gel-purified, cloned, and sequenced using the same primers. All DNA sequence queries were performed using the alignment tool in CLC Sequence Viewer 6.8.

\section{Chromosomal analysis}

The fibroblasts with the MSTN gene knock out were cultured on $60-\mathrm{mm}$ plastic culture dishes at a density of approximately $80 \%$ confluence. Colcemid (Invitrogen) was added to the culture medium at a final concentration of $0.2 \mu \mathrm{g} / \mathrm{mL}$. After a 3-4-h incubation, the cells were harvested, centrifuged, and treated with hypotonic solution $(40 \mathrm{mM} \mathrm{KCl})$ for $20 \mathrm{~min}$. A few drops of methanol and acetic acid $(3: 1, \mathrm{v} / \mathrm{v})$ were added to the hypertonically treated cell suspension and mixed by inversion. The cells were centrifuged, resuspended in a small quantity 
of mix, and spotted onto clean slides. After preparation of chromosome spreads, the karyotypes of cells were analyzed by Giemsa (Sigma) banding and at least 40 metaphase spreads were counted using a microscope.

\section{RESULTS}

\section{Promoter trap vector for knocking out the MSTN gene}

The targeting vector, PIII-MSTN (Figure 1), was successfully constructed using a promoter-trapping strategy for efficiently targeting the bovine MSTN gene (Bradley et al., 2004). Rather than using an internal ribosomal entry site sequence, which is typically used to drive the expression of a reporter gene in gene trapping, we used the EGFP sequence without a promoter for trapping of the MSTN promoter. The targeting vector PIII-MSTN was constructed by inserting 2 homology arms into the PIII construct, which contained a 1-kb EGFP gene sequence without a promoter and a $4.6-\mathrm{kb}$ neo cassette with a phosphoglycerate kinase promoter and polyA signal. The $E G F P$ sequence was located downstream of the initiation codon of the MSTN gene ATG in the 5'-homologous arm. To reduce background expression, we shortened the 5'-homologous arm to $1.5 \mathrm{~kb}$. In addition, PIII-MSTN carried a neo cassette so that gene-targeting events were detectable using $\mathrm{NeO}^{\mathrm{R}}$ expression and $\mathrm{G} 418$ selection.

\section{Comparison of gene transfection in bovine fetal fibroblasts}

The percentage of GFP-positive cells transfected with the linearized EGFP-C1 plasmid by electroporation was $32.90 \%$; using lipofection, this value was $35.27 \%$ (Figure $3 \mathrm{a}$ and b). There were no statistically significant differences between electroporation and lipofection in the transfection of EGFP-C1. However, the morphology of cells after electroporation was superior to that after lipofection (Figure 3c).

\section{Knockout of the MSTN gene with a promoter-trap vector}

According to our results, electroporation resulted in better cell morphology and similar efficiency compared with lipofection. In addition, because electroporation introduces DNA into cells in a naked form that can easily participate in homologous recombination (Potter and Heller, 2011), to assess the performance of the vector PIII-MSTN in the knockout and production of transgenic cells, we introduced PIII-MSTN into $3.4 \times 10^{7}$ bovine primary fetal fibroblasts by electroporation. A total of 104 resistant clones were obtained after G418 selection. Among them, 20 GFP-expressing cell clones were observed under fluorescence microscopy (Figure 4a). Next, we analyzed gene trap insertions within the GFP-expressing clones by 5'-arm PCR. Six cell clones showed the expected PCR-positive band of $2410 \mathrm{bp}$, while most clones did not have this band (Figure $4 \mathrm{~b}$ ). We further confirmed recombination of the PIIIMSTN vector in PCR-positive single cell clones by DNA sequencing and the alignment tool for comparison with the wild-type sequence of the targeted MSTN locus. Only 1 clone (clone 11) contained the exact $M S T N$ short homology arm and a part of the EGFP sequence (Figure $4 c)$. Of the GFP-expressing clones, 5\% (1/20) matched the reported transcribed sequences (Table 1). Chromosomal analysis indicated that cells of clone 11 had normal karyotypes (Figure $4 \mathrm{~d}$ ) and could be used as donors for somatic cell nuclear transfer. 
a
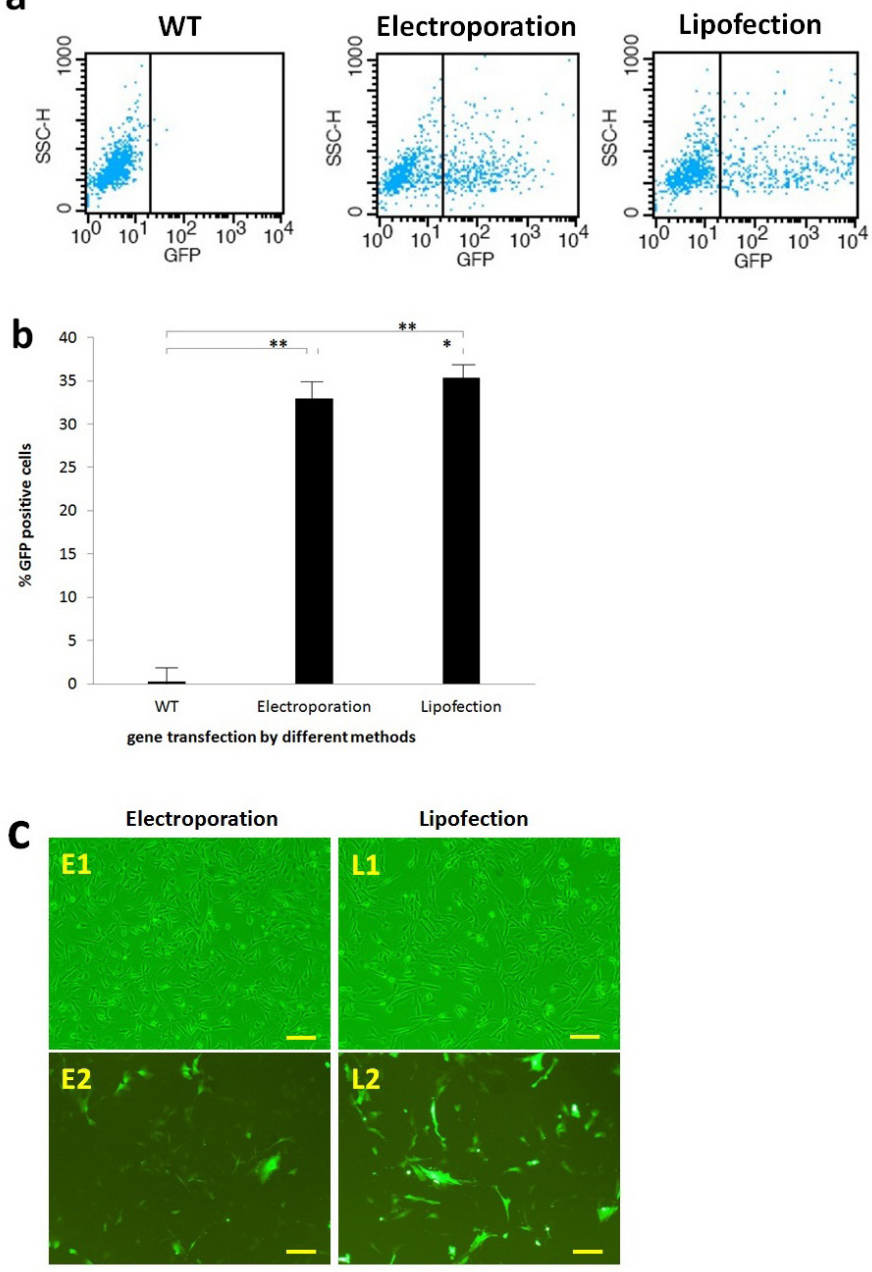

Figure 3. Transfection efficiency of EGFP-C1 into bovine fetal fibroblasts by electroporation and lipofection. a. b. Flow cytometric analysis of EGFP-C1 expression in bovine fetal fibroblasts $48 \mathrm{~h}$ after transfection by electroporation or lipofection. After transfection, there were significantly more GFP-positive cells than wild-type (WT) cells, but no statistically significant differences were observed between electroporation and lipofection. Significant differences between all groups were identified using analysis of variance and were characterized on a pairwise basis using the Welch correct $t$-test $(* \mathrm{P}>0.05, * * \mathrm{P}<0.001)$. c. Fluorescence microscopy images of EGFP-C1 expression in bovine fetal fibroblasts $48 \mathrm{~h}$ after transfection by electroporation or lipofection. Scale bar $=100 \mu \mathrm{m}$. The experiment was repeated 3 times with similar results.

\section{Knockout of the MSTN gene with ZFNs plasmids}

Knockout of the bovine MSTN gene using ZFN plasmids was carried out synchronously in our lab. To mutate the second exon of the MSTN gene, 2 pairs of ZFN plasmids were assembled and transfected into bovine fetal fibroblasts. Nine of the 174 clones (5.17\%) were targeted by ZFNs-MSTN I and 4 of 140 clones (2.86\%) were mutated by ZFNs-MSTN II (mutated sequence not shown). 

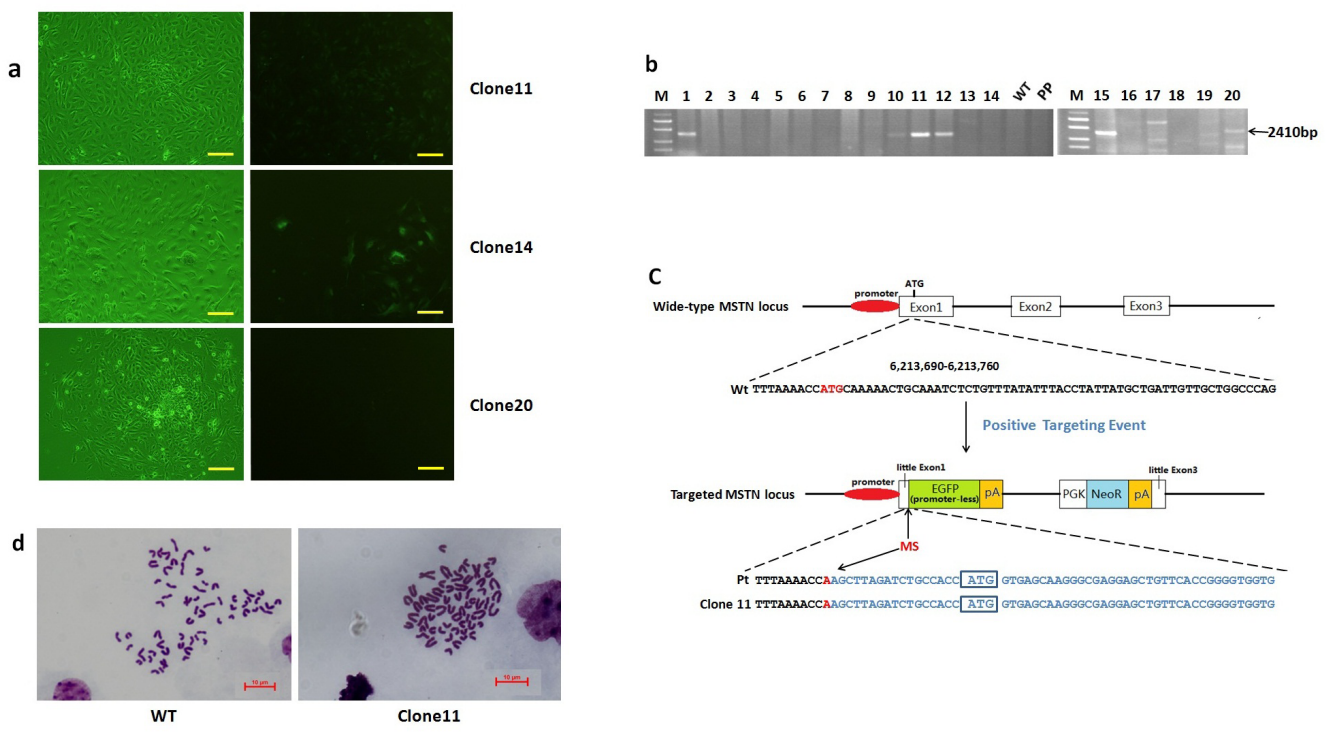

Figure 4. Results of knockout of the $M S T N$ gene with a promoter trap vector. a. GFP expression in transgenic bovine cell clones was observed by fluorescence microscopy after PIII-MSTN transfection and G418 selection. Phase contrast (left) and fluorescent (right) images are shown for 3 of 20 clones. Scale bar $=100 \mu \mathrm{m}$. b. The 5'-arm PCR result of PIII-MSTN insertions within GFP-expressing clones. Primers MF and MR were used to amplify the targeted alleles (see Figure 1 for location of primers). The positive band was $2410 \mathrm{bp}$ in length. The number on the top of the panel represents the clone number (WT = wild-type cells; PP $=$ PIII-MSTN plasmid; $M=\lambda$-EcoT14I DNA marker). c. Alignment results of clone 11 compared to the sequence of targeted MSTN locus across the 5'-homologous arm. The MSTN sequence of clone 11 was amplified from genomic DNA of cell clone 11 by PCR. The sequence of the targeted MSTN locus was predicted from the trap conversion strategy of PIII-MSTN vector (WT = sequence of wild-type $M S T N$ gene; $\mathrm{Pt}$ = sequence of positive targeted MSTN gene). The MSTN sequence after mutation site (MS) was replaced by the marker gene GFP containing ATG (blue boxes). d. Chromosomal analysis. Cells of clone 11 had a normal karyotype of 60 chromosomes as did wild-type cells (WT). Scale bar $=10 \mu \mathrm{m}$.

Table 1. Targeting efficiency comparison between promoter trap vector and ZFNs.

\begin{tabular}{lcccc}
\hline Transformation plasmids & $\begin{array}{c}\text { No. of cells used } \\
\text { for transformation }^{\mathrm{a}}\end{array}$ & $\begin{array}{c}\text { No. of clones picked } \\
\text { up following selection }\end{array}$ & $\begin{array}{c}\text { No. of successful } \\
\text { targeting clones }\end{array}$ & Targeting efficiency (\%) \\
\hline PIII-MSTN & $3.4 \times 10^{7}$ & 20 & 1 & $5.00 \%(1 / 20)$ \\
ZFNs-MSTN I & $1.2 \times 10^{5}$ & 174 & 9 & $5.17 \%(9 / 174)$ \\
ZFNs-MSTN II & $1.2 \times 10^{5}$ & 140 & 4 & $2.86 \%(4 / 140)$ \\
\hline
\end{tabular}

aNumber of cells for PIII-MSTN transformation was $0.4-0.6 \times 10^{7}$ in every experiment of electroporation, so the clones used for mutation analysis were selected from 7 electroporation experiment. Correspondingly, $1.2 \times 10^{5}$ cells were transfected by ZFN-MSTN I or ZFN-MSTN II plasmids in every experiment of lipofection.

\section{DISCUSSION}

Although ZFNs have become one of the most powerful tools for the genomic manipulation of many plants and animals and various types of mammalian cells in basic research, agriculture, and therapeutic applications (Carroll, 2011; Wood et al., 2011), it is unknown whether the efficiency of gene trapping is lower than that of ZFNs for a specific gene. Our results showed that the promoter trap vector for knocking out the bovine myostatin gene was 
similar to the effectiveness of ZFNs. The promoter trap vector was constructed, transfected into bovine fibroblasts, and used to generate an MSTN gene knockout colony. The vector, containing 2 reporter genes and 2 homologous arms, replaced the exons of the MSTN locus, conferring neomycin resistance and allowing for the expression of EGFP after homologous recombination. This suggests that the GFP trapping promoter is an effective strategy for mutating genes in livestock.

Gene-trap vectors typically contain the lac gene as a reporter and the neo gene as a selectable marker; GFP and other genes are also used as reporters to achieve higher sensitivity and to observe the gene-trapping efficiency in living cells (Konishi et al., 2012). In this study, the reporter GFP included its own ATG translation start codon, which replaced the ATG codon of the MSTN gene and incorporated an endogenous internal ribosomal entry site downstream. This is a useful property for detecting targeted cells expressing a trapped gene, as the reporter protein GFP can diffuse throughout the cell body. An efficiency of 19.23\% (20 GFP-expressing clones vs 104 neomycin-resistant clones) demonstrated that promoter-free GFP could insert in-frame with the bovine MSTN gene to produce a fusion protein with green fluorescence.

Another strategy targeting endogenous genes is the use of a polyA trap vector, which includes a strong internal promoter driving the expression of a resistance marker lacking its own polyA signal followed by a splice donor (Yoshida et al., 1995; Salminen et al., 1998). Although the polyA trap design should trap all genes regardless of expression levels, the results of polyA trapping screens have often been below expectations (Zambrowicz et al., 2003; Shigeoka et al., 2005). To overcome this limitation, efforts have been made to combine different promoters and splice donor (SD) signals, insert a synthetic intron within the selectable marker gene, or include the Sleeping Beauty transposon (Lin et al., 2006; Tsakiridis et al., 2009; Song et al., 2012). However, compared with the promoter-trapping strategy, polyA trapping has not been validated in a large-scale gene trap setting. In our experiment, the promoter-free vector PIII-MSTN prevented the GFP marker gene from being expressed by random integration at many loci. GFP-expressing cells would result from rare homologous recombination events. We detected and obtained positive targeting clones by identifying GFP-expressing clones, and the targeting efficiency of 5\% (1 positive targeting clone in 20 GFP-expressing clones) was similar to that obtained by Zhang et al. (2007) and Chen et al. (2013) using other promoter trap vectors. Promoter-free GFP was followed by another selection marker, neo ${ }^{\mathrm{R}}$, which was expressed by the exogenous promoter phosphoglycerate kinase, and screens were designed to achieve a more random distribution of insertions throughout the genome. Therefore, through predigesting selection of cell clones, G418 screening showed that the promoter-free GFP gene was expressed because it had been inserted downstream of other endogenous promoters except for the MSTN promoter.

Since the targeting of COL1A1 in sheep (McCreath et al., 2000), somatic cell nuclear transfer technology has provided an alternative means of cell-mediated transgenesis to circumvent the requirement for embryonic stem cells to generate gene-targeted animals such as sheep (Denning et al., 2001), cattle (Kuroiwa et al., 2009), goat (Liu et al., 2013), and pigs (Dai et al., 2002; Lai et al., 2002). It is technically challenging to produce gene-targeted cell lines as donors with sufficient genetic stability for somatic cell nuclear transfer. Although ZFNs are considered to be promising tools that will radically simplify gene knockout and targeted gene replacement (Carroll, 2011), their design and assembly is labor-intensive and limited by available target sites (Carlson et al., 2012b). It is also difficult to identify all possible off-target cleavage sites. In this study, the number of cells used for ZFN transfection was substantially 
lower than that used for PIII-MSTN plasmids using different transfection methods. Regarding the number of clones screened following selection, ZFN transfection showed more colonies than PIII-MSTN plasmid transfection. However, as targeting efficiency of PIII-MSTN double selection plasmids was similar to ZFN-MSTN I and higher than ZFN-MSTN II, the workload of PCR and sequencing was reduced following selection. Yang et al. (2011) found that only 1 of 3 pairs of ZFNs used to knock out Ppar- $\gamma$ in porcine cells displayed high activity and 5 in 119 screened cell clones were found to carry different mutations in the targeted gene. Here, the efficiency of ZFN-mediated bovine MSTN knockout was similar to that observed in Yang et al. (2011) study, and the targeting efficiency of the trapping vector was not lower. Therefore, promoter trap vectors are effective tools showing the potential to be used in manipulating genomes.

In recent years, other engineered endonucleases, such as TALENs and clustered regularly interspaced short palindromic repeats and their associated systems, have been used for genome alteration (Christian et al., 2010; Li et al., 2011; Cho et al., 2013; Cong et al., 2013; Mali et al., 2013). ZFNs, TALENs, and clustered regularly interspaced short palindromic repeats and their associated systems have been used to generate DNA double-strand breaks at target sites, which are repaired via non-homologous end-joining or homologous recombination. With high-throughput homologous recombination, gene trapping can be combined with these novel strategies as an effective tool for genomic modification.

\section{ACKNOWLEDGMENTS}

Research supported by the National "863" Project of China (\#2009AA10Z111), the National Transgenic Project of China (\#2008ZX08010-001 and \#2011ZX08010-001), and the Inner Mongolia Natural Sciences Foundation (\#2009ZD02). The authors gratefully acknowledge the generous support of the Collaborative Innovation Center for Cardiovascular Disease Translational Medicine, Jiangsu Province, China.

\section{REFERENCES}

Bogdanove AJ and Voytas DF (2011). TAL effectors: customizable proteins for DNA targeting. Science 333: 1843-1846. Bradley PJ, Li N and Boothroyd JC (2004). A GFP-based motif-trap reveals a novel mechanism of targeting for the Toxoplasma ROP4 protein. Mol. Biochem. Parasitol. 137: 111-120.

Carlson DF, Fahrenkrug SC and Hackett PB (2012a). Targeting DNA with fingers and TALENs. Mol. Ther. Nucleic Acids 1: e3.

Carlson DF, Tan W, Lillico SG, Stverakova D, et al. (2012b). Efficient TALEN-mediated gene knockout in livestock. Proc. Natl. Acad. Sci. U. S. A. 109: 17382-17387.

Carroll D (2011). Genome engineering with zinc-finger nucleases. Genetics 188: 773-782.

Chen J, Haverty J, Deng L, Li G, et al. (2013). Identification of a novel endogenous regulatory element in Chinese hamster ovary cells by promoter trap. J. Biotechnol. 167: 255-261.

Cho SW, Kim S, Kim JM and Kim JS (2013). Targeted genome engineering in human cells with the Cas9 RNA-guided endonuclease. Nat. Biotechnol. 31: 230-232.

Christian M, Cermak T, Doyle EL, Schmidt C, et al. (2010). Targeting DNA double-strand breaks with TAL effector nucleases. Genetics 186: 757-761.

Cong L, Ran FA, Cox D, Lin S, et al. (2013). Multiplex genome engineering using CRISPR/Cas systems. Science 339: 819-823.

Dai Y, Vaught TD, Boone J, Chen SH, et al. (2002). Targeted disruption of the alpha1,3-galactosyltransferase gene in cloned pigs. Nat. Biotechnol. 20: 251-255.

Denning C, Burl S, Ainslie A, Bracken J, et al. (2001). Deletion of the alpha(1,3)galactosyl transferase (GGTA1) gene and the prion protein (PrP) gene in sheep. Nat. Biotechnol. 19: 559-562. 
Friedel RH, Plump A, Lu X, Spilker K, et al. (2005). Gene targeting using a promoterless gene trap vector ("targeted trapping") is an efficient method to mutate a large fraction of genes. Proc. Natl. Acad. Sci. U. S. A. 102: 13188-13193.

Friedrich G and Soriano P (1991). Promoter traps in embryonic stem cells: a genetic screen to identify and mutate developmental genes in mice. Genes Dev. 5: 1513-1523.

Hauschild J, Petersen B, Santiago Y, Queisser AL, et al. (2011). Efficient generation of a biallelic knockout in pigs using zinc-finger nucleases. Proc. Natl. Acad. Sci. U. S. A. 108: 12013-12017.

Konishi Y, Karnan S, Takahashi M, Ota A, et al. (2012). A system for the measurement of gene targeting efficiency in human cell lines using an antibiotic resistance-GFP fusion gene. Biotechniques 53: 141-152.

Kuroiwa Y, Kasinathan P, Sathiyaseelan T, Jiao JA, et al. (2009). Antigen-specific human polyclonal antibodies from hyperimmunized cattle. Nat. Biotechnol. 27: 173-181.

Lai L, Kolber-Simonds D, Park KW, Cheong HT, et al. (2002). Production of alpha-1,3-galactosyltransferase knockout pigs by nuclear transfer cloning. Science 295: 1089-1092.

Li T, Huang S, Jiang WZ, Wright D, et al. (2011). TAL nucleases (TALNs): hybrid proteins composed of TAL effectors and FokI DNA-cleavage domain. Nucleic Acids Res. 39: 359-372.

Lin Q, Donahue SL, Moore-Jarrett T, Cao S, et al. (2006). Mutagenesis of diploid mammalian genes by gene entrapment. Nucleic Acids Res. 34: e139.

Liu J, Luo Y, Ge H, Han C, et al. (2013). Anti-bacterial activity of recombinant human beta-defensin-3 secreted in the milk of transgenic goats produced by somatic cell nuclear transfer. PLoS One 8: e65379.

Mali P, Yang L, Esvelt KM, Aach J, et al. (2013). RNA-guided human genome engineering via Cas9. Science 339: 823826.

Marques MM, Thomson AJ, McCreath KJ and McWhir J (2006). Conventional gene targeting protocols lead to loss of targeted cells when applied to a silent gene locus in primary fibroblasts. J. Biotechnol. 125: 185-193.

McCreath KJ, Howcroft J, Campbell KH, Colman A, et al. (2000). Production of gene-targeted sheep by nuclear transfer from cultured somatic cells. Nature 405: 1066-1069.

Miller JC, Tan S, Qiao G, Barlow KA, et al. (2011). A TALE nuclease architecture for efficient genome editing. Nat. Biotechnol. 29: 143-148.

Porteus MH and Baltimore D (2003). Chimeric nucleases stimulate gene targeting in human cells. Science 300: 763.

Potter H and Heller R (2011). Transfection by electroporation. Curr. Protoc. Neurosci. (Appendix) 1: 1E.

Salminen M, Meyer BI and Gruss P (1998). Efficient poly A trap approach allows the capture of genes specifically active in differentiated embryonic stem cells and in mouse embryos. Dev. Dyn. 212: 326-333.

Sedivy JM and Dutriaux A (1999). Gene targeting and somatic cell genetics - a rebirth or a coming of age? Trends Genet. 15: 88-90.

Shigeoka T, Kawaichi M and Ishida Y (2005). Suppression of nonsense-mediated mRNA decay permits unbiased gene trapping in mouse embryonic stem cells. Nucleic Acids Res. 33: e20.

Song G, Li Q, Long Y, Hackett PB, et al. (2012). Effective expression-independent gene trapping and mutagenesis mediated by Sleeping Beauty transposon. J. Genet. Genomics 39: 503-520.

Tsakiridis A, Tzouanacou E, Rahman A, Colby D, et al. (2009). Expression-independent gene trap vectors for random and targeted mutagenesis in embryonic stem cells. Nucleic Acids Res. 37: e129.

Wood AJ, Lo TW, Zeitler B, Pickle CS, et al. (2011). Targeted genome editing across species using ZFNs and TALENs. Science 333: 307.

Yang D, Yang H, Li W, Zhao B, et al. (2011). Generation of PPARgamma mono-allelic knockout pigs via zinc-finger nucleases and nuclear transfer cloning. Cell Res. 21: 979-982.

Yoshida M, Yagi T, Furuta Y, Takayanagi K, et al. (1995). A new strategy of gene trapping in ES cells using 3'RACE. Transgenic Res. 4: 277-287.

Zambrowicz BP, Friedrich GA, Buxton EC, Lilleberg SL, et al. (1998). Disruption and sequence identification of 2,000 genes in mouse embryonic stem cells. Nature 392: 608-611.

Zambrowicz BP, Abuin A, Ramirez-Solis R, Richter LJ, et al. (2003). Wnk1 kinase deficiency lowers blood pressure in mice: a gene-trap screen to identify potential targets for therapeutic intervention. Proc. Natl. Acad. Sci. U. S. A. 100: 14109-14114.

Zhang L, Yang X, An X and Chen Y (2007). Myostatin gene targeting in cultured China Han ovine myoblast cells. Animal 1: 1401-1408.

Zhao L, Liang H, Yun T and Li R (2012). Construction of knock-out vectors for bovine MSTN. Sci. Agric. Sin. 45: 359368. 